\title{
Reversed Phase High Performance Liquid Chromatographic Method for Determination of Sildenafil in Human Plasma and its Application to a Bioequivalence Study
}

\author{
Samah SA, Bendas ER, Abdel-Fattah AA and Hegazy MA* \\ Center of Applied Research and Advanced Studies (CARAS), Faculty of Pharmacy, \\ Cairo University, Egypt
}

*Corresponding author: Maha Hegazy, Analytical supervisor In-Charge, For

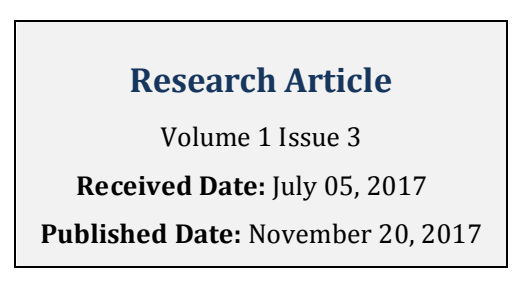

Published Date: November 20, 2017

Biocaras Center, Faculty of Pharmacy, Cairo University, Egypt, Tel: +2 0112887066; E-mail: mahahgazy@yahoo.com

\section{Abstract}

Sildenafil Citrate is a drug used to treat erectile dysfunction and pulmonary arterial hypertension (PAH). A sensitive and specific method based on High Performance Liquid Chromatography (HPLC) was developed and validated for the determination of Sildenafil in human plasma using nabumetone as internal standard. They were extracted from plasma by liquid-liquid extraction and separated isocratically on a C18 $\mu$-Bond pack $5 \mu \mathrm{m}, 150 \times 3.6 \mathrm{~mm}$, Waters, U.S.A, with as mobile phase of acetonitrile: phosphate buffer 0.05M (55: $45 \mathrm{v} / \mathrm{v}$ ), $\mathrm{pH}$ 4.6. Detection was carried out using UV detector at $295 \mathrm{~nm}$. The chromatographic separation was obtained within 10 min and was linear in the concentration range of $25-1200 \mathrm{ng} / \mathrm{mL}(\mathrm{r}=0.9992)$. The method was successfully applied for the bioequivalence study of two tablet formulations of Sildenafil $100 \mathrm{mg}$ after single oral dose administration to 22 healthy human volunteers. Single dose randomized bioequivalence study was done to compare the pharmacokinetic parameters of VIVAG (test, Sildenafil citrate 100mg tablets) versus VIAGRA (references, Sildenafil Citrate 2 X50mg tablets). The $90 \%$ confidence intervals were calculated for the Cmax, AUC $(0-t)$ and AUC $(0-\infty)$, giving values between 88.55-104.06 \% demonstrating the bioequivalence of the two formulations.

Keywords: Sildenafil citrate; $\mathrm{C}_{\max }$ and $\mathrm{T}_{\max }$; Bioequivalence; High Performance liquid chromatography; Liquidliquid extraction; Pharmacokinetics; VIVAG; VIAGRA

\section{Introduction}

Viavag (Sildenafil Citrate) is chemically designated as 1-\{[3-(6, 7-Dihydro-1-methyl-7-oxo-3-propyl-1Hpyrazolo $\quad[4,3-d]$ pyrimidin-5-yl)-4ethoxyphenyl]sulfonyl $\}$-4methyl piperazinium citrate monohydrate), Figure 1 VIVAG is a drug used to treat erectile dysfunction and pulmonary arterial hypertension (PAH).

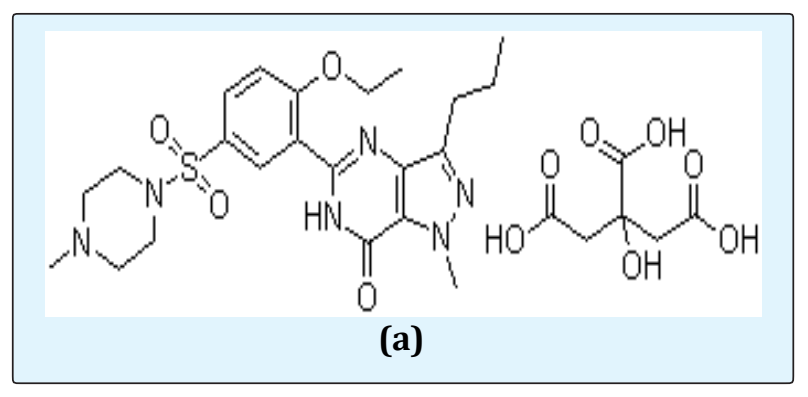




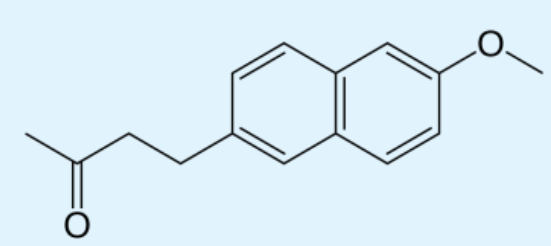

(b)

Figure 1: Structure of Sildenafil Citrate (a) and Nabumetone (IS) (b).

It acts by inhibiting cGMP specific phosphodiesterase type 5 , an enzyme that regulates blood flow in the penis. Since becoming available in 1998, sildenafil has been the prime treatment for erectile dysfunction. The mechanism of action of Sildenafil citrate involves the release of nitric oxide (NO) in the corpus cavernosum of the penis. NO binds to the receptors of the enzyme guanylate cyclase which results in increased levels of cyclic guanosine monophosphate (cGMP), leading to smooth muscle relaxation (vasodilation) of the intimal cushions of the helicine arteries, resulting in increased inflow of blood and an erection [1]. Sildenafil is a potent and selective inhibitor of cGMP specific phosphodiesterase type 5 (PDE5) which is responsible for degradation of cGMP in the corpus cavernosum. The molecular structure of sildenafil is similar to that of cGMP and acts as a competitive binding agent of PDE5 in the corpus cavernosum, resulting in more cGMP and better erections [1].

Without sexual stimulation, and therefore lack of activation of the NO/cGMP system, sildenafil should not cause an erection. Sildenafil is metabolized by liver enzymes and excreted by both the liver and kidneys. If taken with a high-fat meal, absorption is reduced; the time taken to reach the maximum plasma concentration increases by around one hour, and the maximum concentration itself is decreased by nearly one-third. The primary indication of sildenafil is treatment of erectile dysfunction (inability to sustain a satisfactory erection to complete intercourse). It use is now standard treatment for erectile dysfunction in all settings, including diabetes [2]. People on antidepressants may experience sexual dysfunction, either as a result of their illness or as a result of their treatment. A 2003 study showed that sildenafil improved sexual function in men in this situation [3] Following up to earlier reports from 1999 [4] the same researchers found that sildenafil was able to improve sexual function in female patients on antidepressants as well [5] As well as erectile dysfunction, sildenafil citrate is also effective in the rare disease pulmonary arterial hypertension (PAH). It relaxes the arterial wall, leading to decreased pulmonary arterial resistance and pressure. This in turn reduces the workload of the right ventricle of the heart and improves symptoms of right- sided heart failure. Because PDE-5 is primarily distributed within the arterial wall smooth muscle of the lungs and penis, sildenafil acts selectively in both these areas without inducing vasodilatation in other areas of the body. Pfizer submitted an additional registration for sildenafil to the FDA, and sildenafil was approved for this indication in June 2005. The preparation is named Revatio, to avoid confusion with Viagra, and the 20 milligram tablets are white and round. Sildenafil joins bosentan and prostacyclin-based therapies for this condition [6]. Sildenafil has been shown to be useful for the prevention and treatment of High altitude pulmonary edema associated with altitude sickness such as that suffered by mountain climbers $[7,8]$ While this effect has only recently been discovered, sildenafil is already becoming an accepted treatment for this condition, particularly in situations where the standard treatment of rapid descent has been delayed for some reason [9]. The objective of the single dose randomized bioequivalence study was to compare the pharmacokinetic parameters of VIGREX versus VIAGRA.

The reported analytical methods used to determine Sildenafil concentrations in biological samples include the application of HPLC method [10], HPLC coupled with mass dedector (LC-MS) [11-14], HPLC coupled with ESI-MS-MS [15] and determination using micellar electro kinetic chromatography [16].

The aim of the present work was to develop and validate a simple, precise and accurate HPLC method with UV detection, using liquid-liquid extraction, for the pharmacokinetic analysis of Sildenafil in human plasma supporting the bioequivalence study of two pharmaceutical formulations.

\section{Materials and Methods}

\section{Chemicals and Reagents}

The test and reference formulations containing 100 mg of Sildenafil citrate were manufactured by the Pharmaceutical Company Hi-Pharm, Batch 0360909 (Egypt) and Pfizer, Batch 9801 (USA), respectively, within their shelf life period. Sildenafil citrate reference substance (Batch SC06/005/05) was purchased from Cadila Healthcare Limited, Patalganga, India, and Nabumetone (internal standard, I.S.) (Batch L00165) was purchased by Sigma-Aldrich Co, USA. HPLC grade Acetonitrile and methanol were supplied by (Romil Limited, London. United Kingdom \& E.Merk, D-6100 Darmstadt, F.R. Germany). Sodium dihydrogen phospahate, Sigma-Aldrich Co, water used was HPLC grade. The human plasma was obtained from VACSERA (Cairo, Egypt). All solutions were filtered through a 0.45 $\mu \mathrm{m}$ membrane filter (Millipore, Bedford, MA, USA). 


\section{Apparatus and Chromatographic Conditions}

A Shimadzu LC system (Shimadzu, Kyoto, Japan) was used equipped with a SCL-10AVP system controller, LC10 AS pump, DGU-14A degasser, CTO-10 ADVP column oven, SIL-10A auto sampler and a SPD-10A UV-variable wavelength detector. The detector was set at $295 \mathrm{~nm}$ and peak areas were integrated automatically by computer using Lab-solution software program. The experiments were carried out on a reversed phase $\mu$ Bond pack (Waters, USA) analytical column (150mm x $3.6 \mathrm{~mm}$ I.D., with a particle size of $5 \mu \mathrm{m}$ and pore size of $80 \AA$ ) maintained at $35^{\circ} \mathrm{C}$. The LC system was operated isocratically using a mobile phase of was acetonitrile: phosphate buffer $0.05 \mathrm{M}$ (55: $45 \mathrm{v} / \mathrm{v}$ ), $\mathrm{pH} 4.6$. This was filtered through a $0.45 \mu \mathrm{m}$ membrane filter (Millipore, Bedford, MA, USA) and run at a flow-rate of $0.7 \mathrm{~mL} / \mathrm{min}$. The injection volume was $20 \mu \mathrm{L}$ for both standard and samples. The temperature of the auto sampler was kept at $4^{\circ} \mathrm{C}$ and the run-time was $10 \mathrm{~min}$.

\section{Standard Solutions and Calibration Curves}

The primary stock solution of sildenafil was prepared by weighing amount of reference material equivalent to $10 \mathrm{mg}$ sildenafil into a $100 \mathrm{~mL}$ volumetric flask and diluting to volume with methanol, obtaining a concentration of $100 \mu \mathrm{g} / \mathrm{mL}$, and then a secondary stock solution was made to a final dilution of $10 \mu \mathrm{g} / \mathrm{mL}$. Nabumetone (I.S.) stock solution was made at a final concentration of $100 \mu \mathrm{g} / \mathrm{mL}$ using methanol. The prepared stock solutions were stored at $2-8{ }^{\circ} \mathrm{C}$ protected from light. An appropriate aliquots of sildenafil stock solutions were diluted with methanol to obtain the working standards with the concentrations of $0.25,0.50,1.0,3.0,5.0,9.0,12.0 \mu \mathrm{g} / \mathrm{mL}$. Then, $100 \mu \mathrm{L}$ was taken of the working standards to prepare the analytical curves by spiking $900 \mu \mathrm{L}$ blank plasma at concentrations from 0.025 to $1.20 \mu \mathrm{g} / \mathrm{mL}(0.025,0.050$, $0.10,0.30,0.50,0.90,1.20 \mu \mathrm{g} / \mathrm{mL})$. A $50 \mu \mathrm{g} / \mathrm{mL}$ IS working solution was obtained by diluting the stock solution of nabumetone with methanol. The quality control (QC) samples were prepared in blank plasma at concentrations of 0.075 (low), 0.60 (medium) and $1.0 \mu \mathrm{g} / \mathrm{mL}$ (high), and then divided in aliquots that were stored at $-20 \stackrel{\circ}{\circ}$ until analysis. The spiked plasma samples (standards and quality controls) were extracted on each analytical batch along with the unknown samples.

\section{Plasma Extraction}

For the analysis of sildenafil, $1000 \mu \mathrm{L}$ of each plasma sample was transferred to a $15 \mathrm{~mL}$ glass tube, followed by addition of $100 \mu \mathrm{L}$ of IS solution $(50 \mu \mathrm{g} / \mathrm{mL})$. All samples were mixed by vortex agitation for $30 \mathrm{~s}$. Then, a $4 \mathrm{~mL}$ aliquot of extraction solvent, di-ethyl ether, was added using Dispensette Organic (Brand $\mathrm{GmbH}$, Wertheim, Germany) and vortex-mixed for 90s. The tubes were centrifuged for $15 \mathrm{~min}$ at $2700 \mathrm{rpm}$ and the organic layer was filtered through a Millex GV $0.45 \mu \mathrm{m}$ filter unit (Millipore, Bedford, MA, USA) into $15 \mathrm{~mL}$ conical tubes and evaporated under nitrogen stream while immersed in a $40^{\circ} \mathrm{C}$ water bath. The residues were reconstituted with $500 \mu \mathrm{L}$ of mobile phase. The samples were transferred to auto sampler vials and 20 $\mu \mathrm{L}$ was injected into the LC system.

\section{Validation of the Bioanalytical Method}

The method was validated by the determination of the following parameters: specificity, linearity, range, recovery, accuracy, precision, lower limit of quantitation (LLOQ) and stability studies, following the bioanalytical method validation guidelines $[17,18]$.

Specificity: Specificity was assessed using six blank human plasma samples, randomly selected, from different sources (including haemolysed and lipemic plasma), that were subjected to the extraction procedure and chromatographed to determine the extent to which endogenous plasma components could interfere in the analysis of sildenafil or the IS. The results were compared to a solution containing $0.025 \mu \mathrm{g} / \mathrm{mL}$ of sildenafil.

Linearity and Range: The analytical curves were constructed from a blank sample (plasma sample processed without IS), a zero sample (plasma processed with IS) and eight concentrations of sildenafil, including the LLOQ, ranging from 0.025 to $1.20 \mu \mathrm{g} / \mathrm{mL}$. The sildenafil/ IS peak area ratios were plotted against the respective standard concentrations to obtain the graph and the linearity evaluated by the least square regression analysis. The acceptance criteria for each calculated standard concentration was not more than $15 \%$ deviation from the nominal value, except for the LLOQ which was set at $20 \%$.

Recovery: The recovery was evaluated by the mean of the response of three concentrations of sildenafil $(0.075,0.60$ and $1.0 \mu \mathrm{g} / \mathrm{mL})$, dividing the mean of the extracted sample by the mean of the unextracted sample (spiked with the extracted blank plasma residues) at the same concentration level.

Precision and accuracy: To evaluate the inter-day precision and accuracy, QC samples were analysed together with one independent analytical standard curve for 3 days, while intra-day precision and accuracy were evaluated through analysis of the QC samples in six replicates in the same day. Inter- and intra-day precision were expressed as relative standard deviation (RSD). The evaluation of precision and accuracy was based on the criteria [18] that the RSD of each concentration level should be within $\pm 15 \%$ of the nominal concentration. 
Limit of Quantitation: The lowest standard concentration on the analytical curve should be accepted as the limit of quantitation if the following conditions are met: the analyte response at the LLOQ should be at least five times the response compared to blank response and analyte peak (response) should be identifiable, discrete and reproducible with a precision of $20 \%$ and accuracy of $80-120 \%$. The limit of detection (LOD) was defined by the concentration with a signalto-noise ratio of 3 .

Stability Studies: The stability of sildenafil in human plasma was evaluated after each storage period and related to the initial concentration as zero cycle (samples that were freshly prepared and processed immediately). The samples were considered stable if the deviation (expressed as percentage bias) from the zero cycle was within $\pm 15 \%$. The freeze-thaw stability of sildenafil was determined at low, medium and high QC samples $(n=3)$ over three freeze-thaw cycles within 3 days. In each cycle, the frozen plasma samples were thawed at room temperature for $2 \mathrm{~h}$ and refrozen for 24 h. After completion of each cycle the samples were analyzed and the results compared with that of zero cycle. The short term stability was evaluated using three aliquots each of the low, medium and high unprocessed QC samples kept at room temperature $\left(25 \pm 5^{\circ} \mathrm{C}\right)$ for 24 $\mathrm{h}$, and then analysed. For the processed sample stability, three aliquots each one of the low, medium and high QC samples were processed and placed into the auto sampler at $10^{\circ} \mathrm{C}$ and analysed after $100 \mathrm{~h}$. For the long term stability analysis of sildenafil, three aliquots of each QC samples were frozen at $-20^{\circ} \mathrm{C}$ for 50 days and then analysed.

Bioequivalence Study: The study was an open, randomized, two period crossover design with a oneweek washout interval between the doses. Twenty two male healthy volunteers aged between 21 and 45 years and within $15 \%$ of the ideal body weight were selected by clinical evaluation and laboratory tests $[19,20]$. The clinical protocol was approved by the local Ethic Committee and the volunteers given written informed agreement to participate in the study. During each period, a single oral dose of sildenafil (100 mg) was given with $300 \mathrm{~mL}$ of water after an overnight fast of at least $10 \mathrm{~h}$. The dose chosen is clinically accepted and it was expected to produce measurable plasma levels for a sufficient portion of the terminal elimination phase. Blood samples were collected at $0 \mathrm{~h}$ (pre-dose) and at: 5min, 0.25, 0.5, 0.75, 1, 1.5, 2, 3, 4, 6, 8, 10 and $24 \mathrm{~h}$ post dosing. The samples were immediately centrifuged, the plasma separated and kept frozen at $-20^{\circ} \mathrm{C}$ into labeled cryogenic tubes until assayed.

Pharmacokinetics and statistical analysis: The pharmacokinetic parameters of sildenafil, namely: the maximum plasma concentration $(\mathrm{Cmax})$ and time point of maximum plasma concentration (Tmax) were obtained directly from the measured data. The area under the sildenafil plasma concentration time curve (AUC $(0-t)$ ) was computed using the linear trapezoidal rule, whereas, the area under the concentration plasma - time curve from time 0 to the infinity (AUC $(0-\infty)$ ) was calculated as the sum of AUC $(0-t)$ and $\mathrm{Ct} / \mathrm{ke}$, where $\mathrm{t}$ was the time of the last measurable concentration $(\mathrm{Ct})$ and ke was the elimination rate constant. The pharmacokinetic parameters of sildenafil were estimated with Kinetica 2000 software and Microsoft Excel 2010. After logarithmic transformation, AUC (0$\infty)$, AUC (0-t) and Cmax values were subjected to analysis of variance (ANOVA), including the terms for subjects, treatment (sequence) and time period. The residuals of which were then tested for normality, as described by Chow \& Liu [21]. For the evaluation of bioequivalence, the point estimates and $90 \%$ confidence interval (C.I.) for the relative difference between the test and reference formulations (test-reference) in each subject were constructed, using the residual mean square error obtained from the multifactorial ANOVA. The bioequivalence between the two formulations was evaluated based on the $90 \%$ C.I. transformed back for the geometric mean ratios of $\mathrm{AUC}(0-\infty), \operatorname{AUC}(0-\mathrm{t})$ and $C$ max, which are recommended within the $80-125 \%$ interval according to the guidelines $[19,20]$.

\section{Results and Discussion}

\section{Method Development}

To obtain the best chromatographic conditions several columns and a number of parameters in composition of mobile phase were examined to provide sufficient selectivity, sensitivity and resolution in a satisfactory separation time. Mobile phases such as potassium phosphate buffer with different $\mathrm{pH}$ values and water were tested. The use of acetonitrile as organic modifier improved the resolution, separation time and peak symmetry. The optimized conditions described for the LC method were used and validated for the analysis of sildenafil in human plasma, due to the capability and application to the bioequivalence study. In order to produce a clean sample and avoid the introduction of non-volatile materials onto the column and LC system, liquid-liquid extraction was used for the sample preparation. Different organic solvents and mixtures were evaluated, including tert-butyl methyl ether, ethyl acetate, diethyl ether and dichloromethane, and tested at different $\mathrm{pH}$ values. The organic solvent, di-ethyl ether was used without $\mathrm{pH}$ adjustment, producing a chromatogram with no interfering peaks in drug-free plasma eluting at the retention times of interest, and also yielding the highest recovery for the analyte and IS compared to the others extraction solvents. Nabumetone was chosen as the IS as it showed a chromatographic behavior similar to that of the 
analyte and was completely resolved from it under the selected conditions. Representative chromatograms of a blank plasma, plasma spiked with sildenafil at $0.025 \mu \mathrm{g} / \mathrm{mL}$ (LLOQ) and plasma obtained from a human healthy volunteer $8 \mathrm{~h}$ after oral dose administration of $100 \mathrm{mg}$ of sildenafil are presented in Figure 2. Typical standard retention times were $3.7 \mathrm{~min}$ for sildenafil and $7.6 \mathrm{~min}$ for IS.

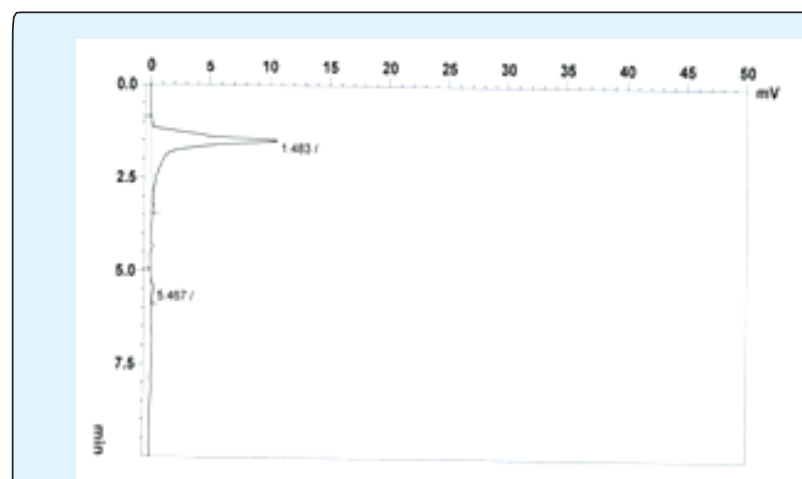

(a)

(b)

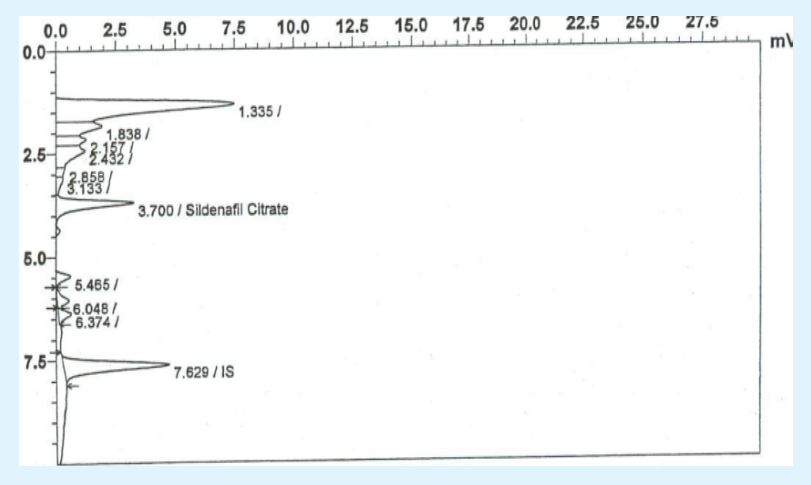

(C)

Figure 2: Representative chromatograms of: (a) Blank plasma sample; (b) Blank plasma sample spiked with sildenafil (LLOQ, $0.025 \mu \mathrm{g} / \mathrm{mL}$ ) and $100 \mu \mathrm{L}$ of nabumetone (IS, $50 \mu \mathrm{g} / \mathrm{mL}$ ); (c) plasma sample of a human healthy volunteer, collected at $8 \mathrm{hr}$ after oral dose administration of sildenafil $100 \mathrm{mg}$ tablet and spiked with $100 \mu \mathrm{L}$ of IS.

\section{Method validation}

Linearity was evaluated by six determinations of eight concentrations in the range of $0.025-1.20 \mu \mathrm{g} / \mathrm{mL}$ The LLOQ was evaluated in an experimental assay and was found to be $0.025 \mu \mathrm{g} / \mathrm{mL}$ with precision and accuracy lower than 20\%. Comparison of the blank and spiked human plasma $(0.025 \mu \mathrm{g} / \mathrm{mL})$ chromatograms indicated that no significant interferences were detected from endogenous substances. Sildenafil in human plasma was directly extracted with di-ethyl ether by liquid-liquid extraction. The mean extraction recoveries for the three concentration levels of the QC samples were $95.57 \%$ for sildenafil and $96.01 \%$ for the IS, nabumetone, showing the method suitability (Table 1 ). The intra-day accuracy of the method was within 94.45 and $101.58 \%$ with a precision of $5.26-8.97 \%$. The inter-day accuracy was within 99.07 and $102.06 \%$ with RSD of $5.33-6.59 \%$ (Table 2). The data show that the method possesses adequate repeatability and reproducibility. Sildenafil was stable in neat plasma for up to $24 \mathrm{~h}$ at room temperature (short-term) and also after three freeze thaw cycles, demonstrating that human plasma samples could be thawed and refrozen without compromising the integrity of the samples. Plasma samples were stable for at least 50 days at -20 ${ }^{\circ} \mathrm{C}$ (long-term). The results demonstrated that extracted samples could be analysed after keeping in the auto sampler for at least $48 \mathrm{~h}$ with acceptable precision and accuracy. The results of stability of sildenafil in human plasma are shown in Table 3.

\begin{tabular}{|c|c|c|}
\hline \multirow{2}{*}{$\begin{array}{c}\text { Nominal } \\
\text { concentration } \\
(\mu \mathrm{g} / \mathrm{mL})\end{array}$} & \multicolumn{2}{|c|}{ Recovery } \\
\hline & $\begin{array}{c}\text { Sildenafil b }{ }^{\text {Mean }} \\
\text { (RSD a \%) }\end{array}$ & $\begin{array}{c}\text { Nabumetone b } \\
\text { Mean (RSD a \%) }\end{array}$ \\
\hline 0.075 & $96.17(2.88)$ & 95.22 (1.91) \\
\hline 0.6 & $96.99(2.00)$ & $96.06(1.63)$ \\
\hline 1 & $93.54(0.75)$ & $96.76(1.11)$ \\
\hline Mean & 95.57 (1.88) & $96.01(0.80)$ \\
\hline
\end{tabular}

a- RSD = Relative standard deviation; b- Mean of six replicates. Table 1: Recovery of sildenafil and nabumetone from human plasma after the extraction procedure.

\begin{tabular}{|c|c|c|c|c|}
\hline $\begin{array}{c}\text { Nominal } \\
\text { concentration } \\
(\boldsymbol{\mu g} / \mathbf{m L})\end{array}$ & \multicolumn{2}{|c|}{ RSD $^{\text {a }} \mathbf{( \% )}$} & \multicolumn{2}{c|}{ Accuracy (\%) } \\
\cline { 2 - 5 } & Intra-day $^{\mathbf{b}}$ & $\begin{array}{c}\text { Inter- } \\
\text { day }^{\mathbf{c}}\end{array}$ & $\begin{array}{l}\text { Intra-day } \\
\mathbf{b}\end{array}$ & $\begin{array}{c}\text { Inter- } \\
\text { day }^{\mathbf{c}}\end{array}$ \\
\hline $\mathbf{0 . 0 7 5}$ & 5.26 & 5.33 & 94.45 & 102.06 \\
$\mathbf{0 . 6 0}$ & 8.97 & 7.48 & 96.64 & 104.03 \\
$\mathbf{1 . 0 0}$ & 5.63 & 6.59 & 101.58 & 99.07 \\
\hline
\end{tabular}

a- RSD = Relative standard deviation; b- Mean of six replicates; $c$ Mean of three days.

Table 2: Inter-day and intra-day precision and accuracy for the determination of sildenafil in human plasma. 


\begin{tabular}{|c|c|c|c|}
\hline Stability conditions & $\begin{array}{c}0.075 \mu \mathrm{g} / \mathrm{mL} \\
\text { Average }^{\mathrm{a}}\left(\text { RSD }^{\mathrm{b}}\right)\end{array}$ & $\begin{array}{c}0.60 \mu \mathrm{g} / \mathrm{mL} \\
\text { Average }^{\text {a }}\left(\text { RSD }^{\mathrm{b}}\right)\end{array}$ & $\begin{array}{c}1.20 \mu \mathrm{g} / \mathrm{mL} \\
\text { Average } \\
\text { a }^{2} \text { RSD }^{\mathrm{b}} \text { ) }\end{array}$ \\
\hline Fresh samples (zero cycle, \%) & $98.09(1.66)$ & $99.35(1.09)$ & $97.45(2.00)$ \\
\hline Freeze-thaw stability (three cycles, $-20^{\circ} \mathrm{C}, \%$ ) & $94.09(2.66)$ & $91.33(4.15)$ & $90.90(2.03)$ \\
\hline Short-term stability (24h, room temperature, \%) & $90.93(1.98)$ & $86.67(3.76)$ & $92.00(3.42)$ \\
\hline Long-term stability ( 50 days, $\left.-20^{\circ} \mathrm{C}, \%\right)$ & $86.70(1.19)$ & $91.18(2.02)$ & $88.40(3.63)$ \\
\hline Post-preparative stability $\left(100 \mathrm{~h}, 10^{\circ} \mathrm{C}, \%\right)$ & $94.62(4.15)$ & $95.39(5.25)$ & $94.17(3.00)$ \\
\hline
\end{tabular}

a - Mean of three replicates; $b$ RSD = Relative standard deviation.

Table 3: Stability of human plasma samples of sildenafil.

\section{Bioequivalence and Statistical Analysis}

The mean pharmacokinetic parameters after a single $100 \mathrm{mg}$ oral dose administration of test and reference products to twenty two healthy volunteers are presented in Tables 4 and 5 . No period effect was observed in the pharmacokinetic parameters studied (data not shown). The curve of the mean sildenafil plasma concentration versus time obtained after a single oral dose of each sildenafil formulation is shown in Figure 3.

At any of the evaluation time, the mean values and the concentrations of sildenafil showed non significant differences between the individual subjects studied after the administration of each of the 2 formulations. The mean Cmax, obtained at 1.64 and $1.61 \mathrm{~h}$, were $0.80 \mu \mathrm{g} / \mathrm{mL}$ and $0.84 \mu \mathrm{g} / \mathrm{mL}$ for test and reference formulations, respectively. Further statistical analysis of pharmacokinetic variables that described the early and total exposure to sildenafil showed point estimates of the geometric means ratios of $C \max , \operatorname{AUC}(0-t)$ and AUC $(0-\infty)$ (sildenafil test vs. sildenafil reference) to be 96.84\% (90\% CIs: 104.06 - 89.61), 95.59\% (90\% CIs: 102.62 - 88.55) and 93.84\% (90 \% CIs: 100.65 -87.03), respectively. For median Tmax values, no significant differences $(P>0.05)$ were found between the two formulations evaluated.

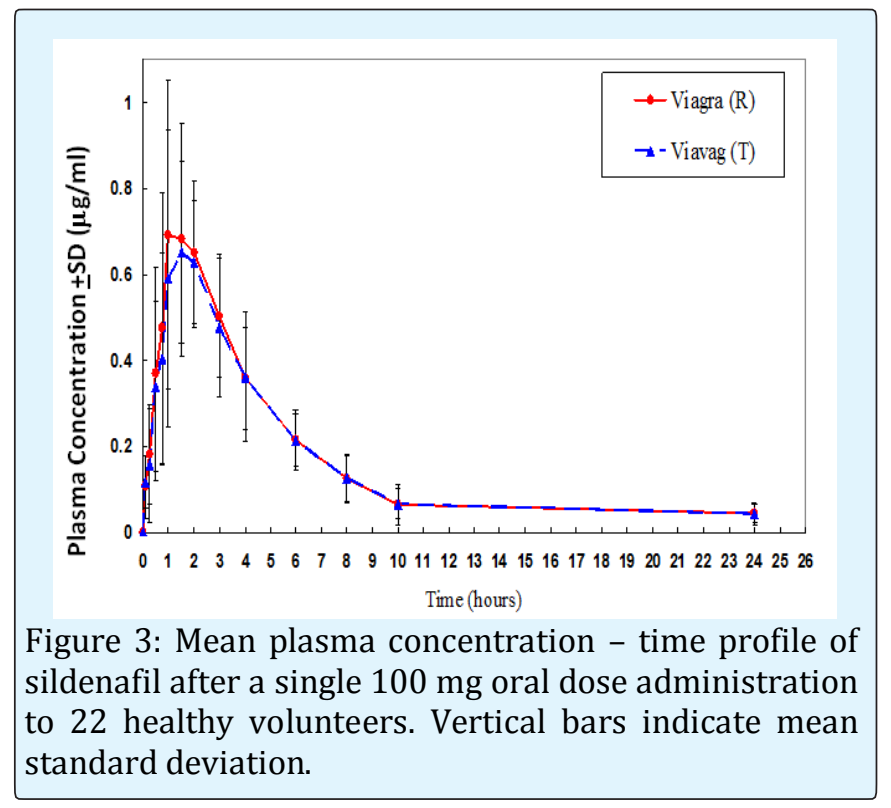

\begin{tabular}{|c|c|c|c|c|}
\hline \multirow{2}{*}{ Parameter } & \multicolumn{2}{|c|}{ Test } & \multicolumn{2}{c|}{ Reference } \\
\cline { 2 - 5 } & Mean & SD $^{\text {a }}$ & Mean & SD $^{\text {a }}$ \\
\hline $\boldsymbol{C m a x}(\boldsymbol{\mu g} / \mathbf{m L})$ & 0.80 & 0.24 & 0.80 & 0.29 \\
\hline $\boldsymbol{T m a x}(\mathbf{h})$ & 1.64 & 0.58 & 1.61 & 0.58 \\
\hline $\boldsymbol{T 1} \mathbf{2} \mathbf{( h )}$ & 3.99 & 3.04 & 4.46 & 3.88 \\
\hline AUC $(\mathbf{0}-\mathbf{t})(\boldsymbol{\mu g} \mathbf{h} / \mathbf{m L})$ & 3.212 & 1.148 & 3.319 & 0.988 \\
\hline AUC $(\mathbf{0}-\boldsymbol{\infty})(\boldsymbol{\mu g} \mathbf{h} / \mathbf{m L})$ & 3.497 & 1.419 & 3.682 & 1.239 \\
\hline
\end{tabular}

$\mathrm{a}-\mathrm{SD}=$ Standard deviation

Table 4: Mean pharmacokinetic parameters for sildenafil, after a single $100 \mathrm{mg}$ oral dose administration of test and reference formulations to 28 healthy volunteers.

\begin{tabular}{|c|c|c|}
\hline Test/Reference & \multicolumn{2}{|c|}{ Parameteric $\mathbf{n = 2 2}$} \\
\hline & Geometric & $\mathbf{9 0 \%}$ CI \\
\hline AUC(0-t) \% ratio & 95.59 & $88.55-102.62$ \\
\hline AUC(0- $\infty)$ \% ratio & 93.84 & $87.03-100.65$ \\
\hline Cmax \% ratio & 96.84 & $89.61-104.06$ \\
\hline
\end{tabular}

a $-\mathrm{RSD}=$ Relative standard deviation.

Table 5: Geometric mean of the individual AUC (0-t), AUC $(0-\infty)$ and $C \max$ ratios (test/reference formulation), the respective $90 \%$ confidence intervals (CI).

\section{Conclusion}

A simple and accurate LC method, with a single step liquid-liquid extraction procedure, has been developed and validated for the determination of sildenafil in human plasma supporting a pharmacokinetic and bioequivalence study. The statistical analysis demonstrated that none of the parameters accepted for drug bioavailability (AUC (0-t), AUC (0- $\infty)$, Cmax) were significantly different between the treatments for the single dose data. Moreover, indicated that the two pharmaceutical products showed similar bioavailability profiles and therefore are considered bioequivalent with regard to the extent and rate of absorption and, interchangeable as well, for clinical and therapeutic purposes.

Acknowledgments: The authors wish to thank HiPharm for Manufactured Pharmaceuticals, Cairo, Egypt for the support and Center of Applied Research and Advanced Studies (CARAS). 


\section{Bioequivalence \& Bioavailability International Journal}

\section{References}

1. Webb DJ, Freestone S, Allen MJ, Muirhead GJ (1999) Sildenafil citrate and blood-pressure-lowering drugs: results of drug interaction studies with an organic nitrate and a calcium antagonist. Am. J. Cardiol 83(5A): 21C-28C.

2. Vardi M, Nini A (2007) Phosphodiesterase inhibitors for erectile dysfunction in patients with diabetes mellitus. Cochrane Database Syst Rev 24(1): CD002187.

3. Nurnberg HG, Hensley PL, Gelenberg AJ, Fava M, Lauriello J, et al. (2003) Treatment of antidepressant-associated sexual dysfunction with sildenafil: a randomized controlled trial. JAMA 289(1): 56-64.

4. Nurnberg HG, Hensley PL, Lauriello J, Parker LM, Keith SJ (1999) Sildenafil for women patients with antidepressant-induced sexual dysfunction. Psychiatr Serv 50(8): 1076-1078.

5. Nurnberg HG, Hensley PL, Heiman JR, Croft HA, Debattista C, et al. (2008) Sildenafil treatment of women with antidepressant-associated sexual dysfunction: a randomized controlled trial. JAMA 300 (4): 395-404.

6. FDA (2005) Approves Pfizer's Revatio as Treatment for Pulmonary Arterial Hypertension.

7. Richalet JP, Gratadour P, Robach P, et al. (2005) Sildenafil inhibits altitude-induced hypoxemia and pulmonary hypertension. Am J Respir Crit Care Med 171(3): 275-281.

8. Perimenis P (2005) Expert Opin Pharmacother 6(5): 835-837.

9. Fagenholz PJ, Gutman JA, Murray AF, Harris NS (2007) Treatment of high altitude pulmonary edema at 4240 m in Nepal. Med. Biol 8(2): 139-146.

10. Ming TH, An BW, Geng CY, Angel H, Hsiu OH (2003) Journal of Chromatography B 791(1-2): 255-262.

11. Vos RM, Chahbouni A, Sinjewel A, Swart EL (2008) Journal of Chromatography B 876(2): 283-287.
12. Pistos C, Papoutsis I, Dona A, Stefanidou M Athanaselis S, et al. (2008) Off-line HPLC method combined to LC-MS for the determination of sildenafil and its active metabolite in post-mortem human blood according to confirmation criteria. Forensic Science International 178(2-3): 192-198.

13. Tracqui A, Ludes (2003) Determination of Blood Cyanide by HPLC-MS. Journal of Analytical Toxicology 27 (2): 88-94.

14. Eerkes A, Addison T, Naidong W (2002) Journal of Chromatography B 768(2): 277-284.

15. Challa BR, Awen BZ, Chandu BR, Khagga M, Bannoth CK, et al. (2010) Sildenafil and Ndesmethyl sildenafil quantification in human plasma by HPLC coupled with ESI-MS/MS detection: Application to bioequivalence study. Anal Methods 8: 1043-1050.

16. Berzas Nevado JJ, Rodríguez Flores J, Castañeda Peñalvo G, Rodríguez Fariñas N (2001) Micellar electro kinetic capillary chromatography for the determination of Viagra and its metabolite (UK103,320) in human serum. Journal of Chromatography A 22(10): 2004-2009.

17. ANVISA (2003) D.O.U. Diário Oficial da União, Poder Executivo,. Guia para Validação de Métodos Analíticos e Bioanalíticos.

18. FDA (2001) Center for Drug Evaluation and Research, US Department of Health and Human Services, Guidance for Industry, Bioanalytical Method Validation.

19. ANVISA (2006) DOU Diário Oficial da União, Poder Executivo. Guia para Provas de Biodisponibilidade Relativa/ Bioequivalência de Medicamentos.

20. FDA (2002) Center for Drug Evaluation and Research, US Department of Health and Human Services, Guidance for Industry, Bioavailability and Bioequivalence Studies for Orally Administered Drug Products - General Considerations.

21. Chow SC, Liu JP (2000) Design and Analysis of Bioavailability and Bioequivalence Studies. $2^{\text {nd }}$ (Edn.) Marcel Dekker, New York, pp: 760. 